\title{
On the Computation of Extinction Time for Some Nonlinear Parabolic Equations
}

\author{
Kossadoum Ngarmadji ${ }^{1}$, Siniki Ndeuzoumbet ${ }^{2}$, Hilaire Nkounkou ${ }^{3}$, Benjamin Mampassi ${ }^{4}$ \\ ${ }^{1}$ University of N'Djamena, N'Djamena, Chad \\ ${ }^{2}$ University of Moundou, Moundou, Chad \\ ${ }^{3}$ Marien Ngouabi University, Brazzaville, Congo \\ ${ }^{4}$ Cheikh Anta Diop University, Dakar, Senegal \\ Email: kossbri@yahoo.fr, siniki_ndeuzoumbet@yahoo.fr, hnkounkou@yahoo.fr, mampassi@yahoo.fr
}

Received 16 March 2015; accepted 6 May 2015; published 7 May 2015

Copyright (C) 2015 by authors and Scientific Research Publishing Inc.

This work is licensed under the Creative Commons Attribution International License (CC BY).

http://creativecommons.org/licenses/by/4.0/

(c) (i) Open Access

\begin{abstract}
The phenomenon of extinction is an important property of solutions for many evolutionary equations. In this paper, a numerical simulation for computing the extinction time of nonnegative solutions for some nonlinear parabolic equations on general domains is presented. The solution algorithm utilizes the Donor-cell scheme in space and Euler's method in time. Finally, we will give some numerical experiments to illustrate our algorithm.
\end{abstract}

Keywords

Nonlinear Parabolic Equations, Donor-Cell Scheme, Numerical Extinction Time, General Domains

\section{Introduction}

There is a large number of nonlinear partial differential equations of parabolic type whose solutions for given initial data become identically nulle in finite time $T$. Such a phenomenon is called extinction and $T$ is called the extinction time. For certain problems, the extinction time can be computed explicitly, but in many cases one can only know the existence of extinction time.

Since the appearance of the pioneering work of Kalashnikov [1], extinction phenomenon in nonlinear parabolic equations has been studied extensively by many authors [2] [3]. Particular emphasis has been placed on the question as to the existence of extinction time [4]-[7].

Generally speaking, it is difficult to simulate extinction phenomenon accurately on general domains. Indeed, it is not at all clear if features of such a phenomenon as extinction can be well reflected in the discretized equation which approximates the original equation. In [8]-[11], some numerical schemes have been used to

How to cite this paper: Ngarmadji, K., Ndeuzoumbet, S., Nkounkou, H. and Mampassi, B. (2015) On the Computation of Extinction Time for Some Nonlinear Parabolic Equations. Applied Mathematics, 6, 754-763.

http://dx.doi.org/10.4236/am.2015.65071 
study the extinction phenomenon of solutions for some nonlinear parabolic equations.

In this work, we propose a numerical algorithm for computing the extinction time for nonnegative solutions of some nonlinear parabolic equations. Our motivation is to reproduce the extinction phenomenon of some nonlinear parabolic equations on general domains.

This paper is organized as follows. In the next section, we present the problem model and some theoretical results. A discretization of this problem is derived in Section 3, while numerical experiments are reported in Section 4 and Section 5 is devoted to concluding remarks.

\section{The Model Problem}

In this work, we are concerned with the following initial-boundary value problem:

$$
\begin{aligned}
& \left.\frac{\partial u}{\partial t}=\Delta \phi(u)-F(u) \text { in } \Omega \times\right] 0, \infty[, \\
& u=0 \quad \text { on } \quad \partial \Omega \times[0, \infty[, \\
& u(0)=u_{0}>0 \text { in } \bar{\Omega}=\Omega \cup \partial \Omega,
\end{aligned}
$$

where $\Omega$ is a $\mathbb{R}^{2}$ bounded domain with boundary $\partial \Omega, u_{0}, \phi$ and $F$, being given functions.

Furthermore, for any $t>0$ and for all $u$ defined in $\Omega \times] 0, \infty[$, we will set $u(t)(x)=u(t, x), x \in \Omega$.

Nonlinear parabolic equations of type (1) appear in various applications. In particular they are used to describe a phenomenon of thermal propagation in an absorptive medium where $u$ stands for temperature [5]. In other applications, $u$ is a concentration and the process is described as diffusion with absorption.

The problem of determining necessary and sufficient conditions on the functions $\phi$ and $F$ which ensure the existence of an extinction time for solutions of (1)-(3) has been considered by several authors [1] [6] [7] [12].

In this section, we state the following result:

Theorem 1 Assume that $u \in C^{1}\left(0, \infty ; L^{2}(\Omega)\right)$ is a nonnegative solution of the problem (1)-(3) where $\phi$ and $F$ are nondecreasing, nonnegative derivatives functions and if $F(0)=0$, then

$$
\lim _{t \rightarrow \infty} \int_{\Omega} \int_{0}^{u(t)} F(s) \mathrm{d} s=0
$$

Proof: First, let us set $G(u(t))=\int_{0}^{u(t)} F(s) \mathrm{d} s$. We have

$$
\frac{\mathrm{d}}{\mathrm{d} t} \int_{\Omega} G(u)=\int_{\Omega} F(u) \frac{\mathrm{d} u}{\mathrm{~d} t} .
$$

Multiplying Equation (1) by $F(u)$ and integrating over $\Omega$ it follows

$$
\int_{\Omega} F(u) \frac{\partial u}{\partial t}=\int_{\Omega} F(u) \Delta \phi(u)-\int_{\Omega}(F(u))^{2} .
$$

On one hand thanks to regularity of functions $F, \phi$ and $u$, we can write

$$
\int_{\Omega} F(u) \Delta \phi(u)=\int_{\partial \Omega} F(u) \nabla \phi(u) \cdot n \mathrm{~d} s-\int_{\Omega} \nabla F(u) \cdot \nabla \phi(u)
$$

where $n$ is the unit outward to $\partial \Omega, \mathrm{d} s$ denotes an element of surface area, since $u$ vanishes on $\partial \Omega$ and $F(0)=0$, we have $\int_{\partial \Omega} F(u) \Delta \phi(u) \cdot n \mathrm{~d} s=0$, hence

$$
\int_{\Omega} F(u) \Delta \phi(u)=-\int_{\Omega} \nabla F(u) \cdot \nabla \phi(u) .
$$

From (ii) and (iii), we deduce

$$
\begin{aligned}
\int_{\Omega} F(u) \frac{\partial u}{\partial t} & =-\int_{\Omega} F^{\prime}(u) \phi^{\prime}(u)|\nabla u|^{2}-\int_{\Omega}(F(u))^{2} \\
& \leq-\int_{\Omega}(F(u))^{2}
\end{aligned}
$$


Since $F^{\prime}(u) \geq 0$ and $\phi^{\prime}(u) \geq 0$, and according to $(i)$, we obtain

$$
\frac{\mathrm{d}}{\mathrm{d} t} \int_{\Omega} G(u) \leq-\int_{\Omega}(F(u))^{2} .
$$

On the other hand, multiplying Equation (1) by $u$ yields

$$
\int_{\Omega} u \frac{\partial u}{\partial t}=-\int_{\Omega} \phi^{\prime}(u)|\nabla u|^{2}-\int_{\Omega} u F(u) \leq 0,
$$

which we rewrite as

$$
\frac{\mathrm{d}}{\mathrm{d} t} \int_{\Omega} u^{2} \leq 0,
$$

then the application $t \mapsto \int_{\Omega} u^{2}$ is decreased in $\mathbb{R}^{+}$. It then follows

$$
\int_{\Omega}(u(t))^{2} \leq \int_{\Omega}(u(0))^{2}=\int_{\Omega} u_{0}^{2} \equiv M^{2}, \quad \text { for all } t>0 .
$$

On the other hand, the increase of $F$ implies that

$$
G(u)=\int_{0}^{u} F(s) \mathrm{d} s \leq F(u) \times \int_{0}^{u} \mathrm{~d} s=u F(u),
$$

Thus

$$
\int_{\Omega} G(u) \leq \int_{\Omega} u F(u) \leq\|u\|_{L^{2}(\Omega)} \times\|F(u)\|_{L^{2}(\Omega)}
$$

and according to $(v i)$ we obtain

$$
\int_{\Omega} G(u) \leq|M|\|F(u)\|_{L^{2}(\Omega)},
$$

This last inequality implies

$$
-\frac{1}{M^{2}}\left(\int_{\Omega} G(u)\right)^{2} \geq-\int_{\Omega}(F(u))^{2} .
$$

Then considering $(i v)$, we deduced

$$
\frac{\mathrm{d}}{\mathrm{d} t} \int_{\Omega} G(u) \leq-\int_{\Omega}[F(u)]^{2} \leq-\frac{1}{M^{2}}\left(\int_{\Omega} G(u)\right)^{2} .
$$

Setting $w(t)=\int_{\Omega} G(u(t))$, we obtain

$$
w^{\prime}(t) \leq-\frac{1}{M^{2}} w^{2}(t) .
$$

This gives after integrating

$$
0 \leq w(t) \leq \frac{1}{\frac{1}{w(0)}+\frac{t}{M^{2}}} .
$$

Knowing that $w(0)>0$. it follows $w(t) \geq 0$. The passage to the limit allows us to write

$$
0 \leq \lim _{t \rightarrow \infty} w(t) \leq \lim _{t \rightarrow \infty} \frac{1}{\frac{1}{w(0)}+\frac{t}{M^{2}}}=0 .
$$

Finally,

$$
\lim _{t \rightarrow \infty} w(t)=\lim _{t \rightarrow \infty} \int_{\Omega} \int_{0}^{u(t)} F(s) \mathrm{d} s=0 .
$$

In addition to the assumption of increase of $F$ in Theorem 2.1, if we assume that 


$$
F(s) \geq C s^{p}, \forall s>0, \forall p \geq 1,
$$

$C$ is a positive constant.

then the following result is easily shown.

Corollary 1 Suppose that the assumptions of the Theorem 2.1 are satisfied, and if (4) holds for $p \geq 1$, then

$$
\lim _{t \rightarrow \infty}\|u(t)\|_{L^{2}(\Omega)}=0 .
$$

Indeed, for all $u(t)$ solution of (1)-(3), it comes from the assumption (4) that

that gives

$$
C \int_{\Omega} \int_{0}^{u(t)} s^{p} \mathrm{~d} s \leq \int_{\Omega} \int_{0}^{u(t)} F(s) \mathrm{d} s,
$$

$$
\int_{\Omega} u^{p+1}(t) \leq \frac{p+1}{C} \int_{\Omega} \int_{0}^{u(t)} F(s) \mathrm{d} s .
$$

As $p+1 \geq 2$, then

$$
0 \leq \int_{\Omega} u^{2}(t) \leq \frac{p+1}{C} \int_{\Omega} \int_{0}^{u(t)} F(s) \mathrm{d} s
$$

So

$$
0 \leq \lim _{t \rightarrow \infty} \int_{\Omega} u^{2}(t) \leq \frac{p+1}{C} \lim _{t \rightarrow \infty} \int_{\Omega} \int_{0}^{u(t)} F(s) \mathrm{d} s,
$$

and as a consequence of Theorem 2.1

$$
\lim _{t \rightarrow \infty}\|u(t)\|_{L^{2}(\Omega)}=0 .
$$

In summary, under some assumptions we know that all nonnegative solutions of (1)-(3) have extinction time as $t \rightarrow \infty$. We want to determine whether extinction occurs in finite time for any given $\phi$ and $F$.

It is well known that, in general, there is no classical solution to this nonlinear parabolic equation for arbitrary choices of $\phi$ and $F$. However, there are some works dealing with approximation of extinction time for solutions of (1). For example, in [13] a numerical method to approximate the solutions of (1) has been developed in the case $N=1$ and in [14] an algorithm based on splitting technique was derived to compute the extinction time for solutions on a rectangular domain.

In order to determine the extinction time for some $\phi$ and $F$, we will derive in the next section a numerical scheme based on Donor-cell scheme. Given a sufficiently small parameter $\epsilon>0$, we would like to determine the positive real $T_{\epsilon}>0$ such that a solution $u(t)$ of the problem (1)-(3) has to satisfy the above relation

$$
\|u(t)\| \leq \epsilon, \quad \forall t>T_{\epsilon} .
$$

We shall call $T_{\epsilon}$ satisfying (6) as the $\epsilon$-extinction time.

\section{Discretization}

\subsection{Discretization of the Studied Domain}

Let $\Omega \subset \mathbb{R}^{2}$ be a considered domain that we assume to be of irregular shape, we approximate $\Omega$ by a domain $\Omega_{h}$ whose boundary is specified by the set of boundary edges lying on gridlines. We imbed $\Omega_{h}$ in a rectangular domain $\Omega_{R}=[a, b] \times[c, d],(a, b>0)$ of smallest possible size. Given two nonzero integers $i_{\max }$ and $j_{\max }$, we set $\delta x=(b-a) / i_{\max } \delta y=(d-c) / j_{\max }$ and we introduce on $\Omega_{R}$ a grid of step $\delta x$ and $\delta y$ in $x$ and $y$ direction respectively. The set of points $\left(x_{i}, y_{j}\right)$ such that of $x_{i}=a+i \delta x, y_{j}=c+j \delta y$ defines the discretization of $\Omega_{R}$ into $i_{\max } \times j_{\max }$ cells (rectangular subdomains). For all $i=1, \cdots, i_{\max }, j=1, \cdots, j_{\max }$ cell $(i, j)$ occupies the spatial region $\left[x_{i}, x_{i+1}\right] \times\left[y_{j}, y_{j+1}\right]$ and has center the point noted $X_{i, j}=\left(x_{i+1 / 2}, y_{i+1 / 2}\right)$. The cells of $\Omega_{R}$ are then divided into inner cell (which lie completely in $\Omega$ ), external cell (which lie in $\Omega_{R} \backslash \bar{\Omega}$ ) and boundary cells (which lie in a part of $\partial \Omega$ ). The problem model is then solved only in the inner 
cells.

A matrix of size $i_{\max } \times j_{\max }$ gives a description of the discretized domain. For example, consider three sets of indices $I, B$ et $E$ corresponding to the inner, boundary and external cells, we then admit to define the following matrix

$$
C(i, j)= \begin{cases}1, & \text { if }(i, j) \in I, \\ -1, & \text { if }(i, j) \in B \\ 0, & \text { if }(i, j) \in E .\end{cases}
$$

the matrix to identify cell types.

The idea of this numerical treatment of general domains has been suggested by Griebel et al. in [15]. An example of this numerical treatment is illustrated in Figure 1 and its matrix representative is given by the following (8).

$$
C=\left(\begin{array}{cccccccccccc}
0 & 0 & 0 & 0 & 0 & 0 & 0 & 0 & 0 & 0 & 0 & 0 \\
0 & 0 & -1 & -1 & -1 & -1 & -1 & -1 & 0 & 0 & 0 & 0 \\
0 & -1 & 1 & 1 & 1 & 1 & 1 & 1 & 1 & -1 & 0 & 0 \\
0 & -1 & 1 & 1 & 1 & 1 & 1 & 1 & 1 & -1 & 0 & 0 \\
0 & -1 & 1 & 1 & 1 & 1 & 1 & 1 & 1 & 0 & 0 & 0 \\
0 & -1 & 1 & 1 & 1 & 1 & 1 & 1 & -1 & 0 & 0 & 0 \\
0 & -1 & 1 & -1 & -1 & 1 & 1 & 1 & -1 & -1 & 0 & 0 \\
0 & 0 & -1 & 0 & 0 & -1 & 1 & 1 & 1 & 1 & -1 & 0 \\
0 & 0 & 0 & 0 & 0 & 0 & -1 & 1 & 1 & 1 & -1 & 0 \\
0 & 0 & 0 & 0 & 0 & 0 & 0 & -1 & 1 & 1 & -1 & 0 \\
0 & 0 & 0 & 0 & 0 & 0 & 0 & 0 & -1 & -1 & 0 & 0 \\
0 & 0 & 0 & 0 & 0 & 0 & 0 & 0 & 0 & 0 & 0 & 0
\end{array}\right)
$$

\subsection{Spatial Discretization}

First of all, let us give an approximation of the diffusion operator at the point $X_{i, j}$ which we rewrite as

$$
[\Delta \phi(u)]_{X_{i, j}}=\left[\frac{\partial}{\partial x}\left(\phi^{\prime}(u) \frac{\partial u}{\partial x}\right)\right]_{X_{i, j}}+\left[\frac{\partial}{\partial y}\left(\phi^{\prime}(u) \frac{\partial u}{\partial y}\right)\right]_{X_{i, j}}
$$
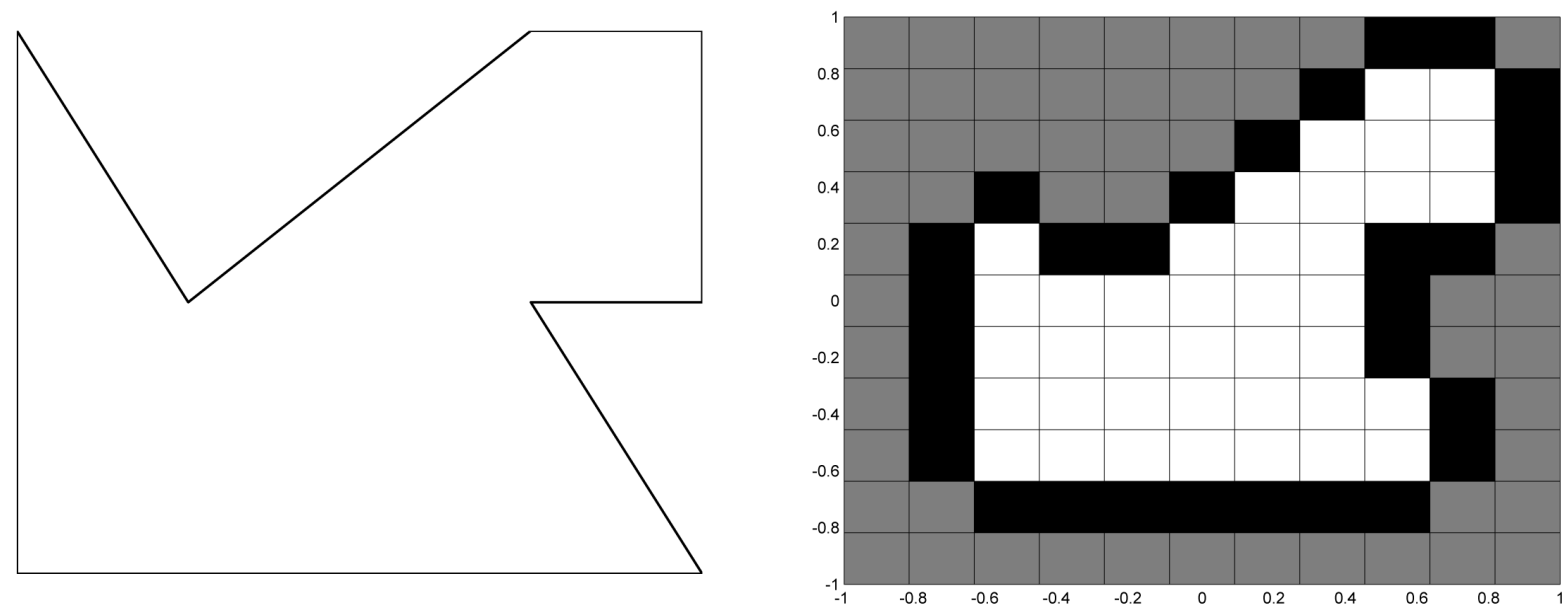

Figure 1. An example of the discretization of a non rectangular domain into cells. 
Let $V_{i, j}$ be the approximation of $\left[\phi^{\prime}(u) \frac{\partial u}{\partial x}\right]$ at the cell center $X_{i, j}$. In the following we do apply a discretization that is similar to the one of Donor-cell scheme where the expression $\left[\phi^{\prime}(u) \frac{\partial u}{\partial x}\right]$ is approached by a progressive finite differences scheme and $\left[\frac{\partial}{\partial x}\left(\phi^{\prime}(u) \frac{\partial u}{\partial x}\right)\right]$ by a central finite differences scheme.

Furthermore, we set $\phi_{i, j}^{\prime}=\phi^{\prime}\left(U_{i, j}\right)$ where $U_{i, j} \approx u\left(X_{i, j}\right)$ and we note

$$
\underline{\Phi}=\left(\phi_{0,0}^{\prime}, \cdots, \phi_{i_{\max }-1,0}^{\prime}, \phi_{0,1}^{\prime}, \cdots, \phi_{i_{\max }-1,1}^{\prime}, \cdots, \phi_{0, j_{\max }-1}^{\prime}, \cdots, \phi_{i_{\max }-1, j_{\max }-1}^{\prime}\right)^{\mathrm{T}}
$$

If $\underline{V}_{x}$ denotes the vector of components $V_{i, j}$ then, one can write

$$
\underline{V}_{x}=\operatorname{diag}(\underline{\Phi}) \times D_{x}^{p} \underline{U}
$$

where $\operatorname{diag}(\underline{\Phi})$ the diagonal matrix whose diagonal is the vector $\Phi$ and $D_{x}^{p}$ denotes forward differentiation matrix the $x$-direction.

On the other hand, denoting by $W_{i, j}$ the approached value of $\left[\frac{\partial}{\partial x}\left(\phi^{\prime}(u) \frac{\partial u}{\partial x}\right)\right]$ at the cell center $X_{i, j}$ and noting by

$$
\underline{W}_{x}=\left(W_{0,0}, \cdots, W_{i_{\max }-1,0}, W_{0,1}, \cdots, W_{i_{\max }-1,1}, \cdots, W_{0, j_{\max }-1}, \cdots, W_{i_{\max }-1, j_{\max }-1}\right)^{\mathrm{T}}
$$

the vector of the value of $\left[\frac{\partial}{\partial x}\left(\phi^{\prime}(u) \frac{\partial u}{\partial x}\right)\right]$ at point $X_{i, j}$, it follows through the central difference scheme, the relation

$$
\underline{W}_{x}=D_{x}^{c} \underline{V}_{x}
$$

which is written by the mean of the Equation (12) as

$$
\underline{W}_{x}=D_{x}^{c} \times \operatorname{diag}(\underline{\Phi}) \times D_{x}^{p} \underline{U},
$$

where $D_{x}^{c}$ denotes central differentiation matrix in the $x$-direction.

Similarly, given $\underline{W}_{y}$, the vector of the approached values of $\left[\frac{\partial}{\partial y}\left(\phi^{\prime}(u) \frac{\partial u}{\partial y}\right)\right]$ at point $X_{i, j}$, we obtain

$$
\underline{W}_{y}=D_{y}^{c} \times \operatorname{diag}(\underline{\Phi}) \times D_{y}^{p} \underline{U},
$$

where $D_{y}^{p}$ denotes forward differentiation matrix in the $y$-direction.

From Equations (13) and (14), we deduce the approximation of the operator $u \mapsto \Delta \phi(u)$ at cell center $X_{i, j}$, in matrix form:

$$
L(\underline{U})=\left(D_{x}^{c} \times \operatorname{diag}(\underline{\Phi}) \times D_{x}^{p}+D_{y}^{c} \times \operatorname{diag}(\underline{\Phi}) \times D_{y}^{p}\right) \underline{U}
$$

where $L(\underline{U})$ is the vector of value of $\Delta \phi(u)$ at points $X_{i, j}$. Thus, we have defined, an approximation operator $L$ to approach the operator $u \mapsto \Delta \phi(u)$.

However, it should be noted that $\underline{\Phi}$ is a vector dependent of $\underline{U}$.

Considering lexicographic numerotation, we note by $\underline{\tilde{U}}(t)$ the vector of the values of $u$ in points $X_{i, j} \in \Omega$ at time $t$. Knowing that $u \equiv 0$ at points $X_{i, j} \in \Omega_{R} \backslash \bar{\Omega}$, and the differentiation matrices $D_{x}^{c}, D_{y}^{c}, D_{x}^{p}, D_{y}^{p}$ to approach the derivative on the set of the points $X_{i, j}$, we can replace respectively by the matrices which are obtained by deleting the rows and columns corresponding to the indices of the points $X_{i, j}$ of $\Omega_{R} \backslash \bar{\Omega}$.

Given the Equation (15), the discrete system approaching the problem (1)-(3) is rewritten by

$$
\frac{\mathrm{d} \underline{\tilde{U}}}{\mathrm{~d} t}=\tilde{D} \times \operatorname{diag}(\underline{\tilde{\Phi}}) \underline{\tilde{U}}-\underline{F}(\underline{\tilde{U}})
$$


where we have set

$$
\tilde{D}=\tilde{D}_{x}^{c} \times \tilde{D}_{x}^{p}+\tilde{D}_{y}^{c} \times \tilde{D}_{y}^{p}
$$

and where $\operatorname{diag}(\underline{\tilde{\Phi}})$ is the matrix obtained of $\operatorname{diag}(\underline{\Phi})$ by deleting the rows and columns corresponding to the indices of the points $X_{i, j}$ of $\Omega_{R} \backslash \bar{\Omega}$ and $\underline{F}(\underline{\tilde{U}})$ is the vector of values of $F\left(u\left(X_{i, j}\right)\right), X_{i, j} \in \Omega$. Furthermore, the initial condition is written

$$
\tilde{U}(0)=\underline{U}_{0}
$$

where $\tilde{U}_{0}$ is the vector obtained from $\underline{U}_{0}$ by deleting the elements corresponding to the indices of the points $X_{i, j}$ of $\Omega_{R} \backslash \bar{\Omega}$

\subsection{Temporal Discretization}

For a time step fixed $\delta t$, we consider the sequence $\left(t_{n}\right)_{n \geq 0}$ defined by $t_{n+1}=t_{n}+\delta t$ et $t_{0}=0$. Then, we denote $\tilde{U}^{n}$ the approximation at time $t_{n}$ of vector $\underline{\tilde{U}}(t)$ solution of (16)-(17). Using the explicit Euler method, the semi-discret scheme is written

$$
\left\{\begin{array}{l}
\underline{\tilde{U}}^{n+1}=\underline{\tilde{U}}^{n}+\delta t \tilde{D} \times \operatorname{diag}\left(\underline{\tilde{\Phi}}^{n}\right) \underline{\tilde{U}}^{n}-\delta t \underline{F}\left(\underline{\tilde{U}}^{n}\right), \\
\underline{\tilde{U}}^{0}=\underline{\tilde{U}}(0),
\end{array}\right.
$$

where we set $\operatorname{diag}\left(\underline{\tilde{\Phi}}^{n}\right)=\operatorname{diag}\left(\underline{\tilde{\Phi}}\left(\underline{\tilde{U}}^{n}\right)\right)$.

It should be noticed that if the time step $\delta t$ is chosen to be little enough, and $F$ satisfied the growth condition (4),

$$
\lim _{n \rightarrow \infty}\left\|\tilde{U}^{n}\right\|=0
$$

Thus the extinction time is obtained using simple itrations process until the stopping criterion

$$
\left\|\tilde{U}^{n}\right\| \leq \epsilon
$$

is satisfied. Here $\epsilon$ is the given tolerance number. The sequence of computations to be performed is summarized as follows

\section{Algorithm 3.1}

1. Read $i_{\max }, j_{\max }, \delta t, \epsilon$ and itermax.

2. Compute $\delta x$ and $\delta y$.

3. Define $\Omega_{R}$ (rectangular) and $\Omega$ (non rectangular) such $\Omega \subset \Omega_{R}$.

4. Compute the matrix $D_{x}^{c}, D_{y}^{c}, D_{x}^{p}, D_{y}^{p}, \tilde{D}$ and $\operatorname{diag}(\underline{\Phi})$.

5. Set $t=0, n=0$.

6. Assign initial value to $\tilde{U}$.

7. Set $\tilde{U}_{\text {old }}=\tilde{U}_{0}$.

8. While $\| \tilde{U}$ old $\|<\epsilon$ and $n<$ itermax, do.

9. Compute $\underline{\tilde{U} \text { new }}$ according to (18) .

10. Set $\tilde{\tilde{U}}$ old $=\underline{\tilde{U}}$ new .

11. $t=t+\delta t, n=n+1$.

End while

\section{Numerical Experiments}

Let $\Omega$ be a bounded domain in $\mathbb{R}^{2}$. Consider the initial value problem

$$
\left.\frac{\partial u}{\partial t}=\Delta u-u^{q}, \quad \text { in } \quad \Omega \times\right] 0, \infty[
$$




$$
\begin{aligned}
& u=0 \quad \text { on } \partial \Omega \times[0, \infty[ \\
& u(0)=u_{0}>0 \text { in } \bar{\Omega}=\Omega \cup \partial \Omega .
\end{aligned}
$$

where $u_{0}$ is the continuous nonnegative function in $\bar{\Omega}$, vanishing on $\partial \Omega$, and $q>0$.

Equation (21) models heat propagation in medium where the solution $u$ stands for temperature.

For our numerical experiments we have consider Figure 2 to be our studied domain and we have use discretization parameters $\delta t=10^{-5}$, and $i_{\max }=j_{\max }=50$.

We would like to numerically estimate the extinction time for solutions of problem (21)-(23) with the initial condition given by

$$
u_{0}(x, y)=1-\left(x^{2}+y^{2}\right)
$$

First, for fixed accurate value $\epsilon_{0}>0$, we estimate the $\mathbb{R}^{N}$-euclidian norm of the sequence $\tilde{U}^{n}$ solution of the numerical scheme (18) for various values of parameters $n$. Table 1 and Figure 3 clearly show that the approximation extinction time can be given by

$$
T_{\epsilon_{0}}=\lim _{n \rightarrow \infty} T_{\epsilon_{0}}^{n}
$$

We can see in Table 1 that this value is approximated by

$$
T_{\epsilon_{0}}=0.61
$$

Also, the extinction process is illustrated by Figure 4 where we can appreciate the numerical solution extinct in a finite time.

\section{Concluding Remarks}

In this paper, a numerical algorithm based on Donor-cell scheme was proposed in order to compute the extinction time for nonnegative solutions of some nonlinear parabolic equations on general domains. We have verified

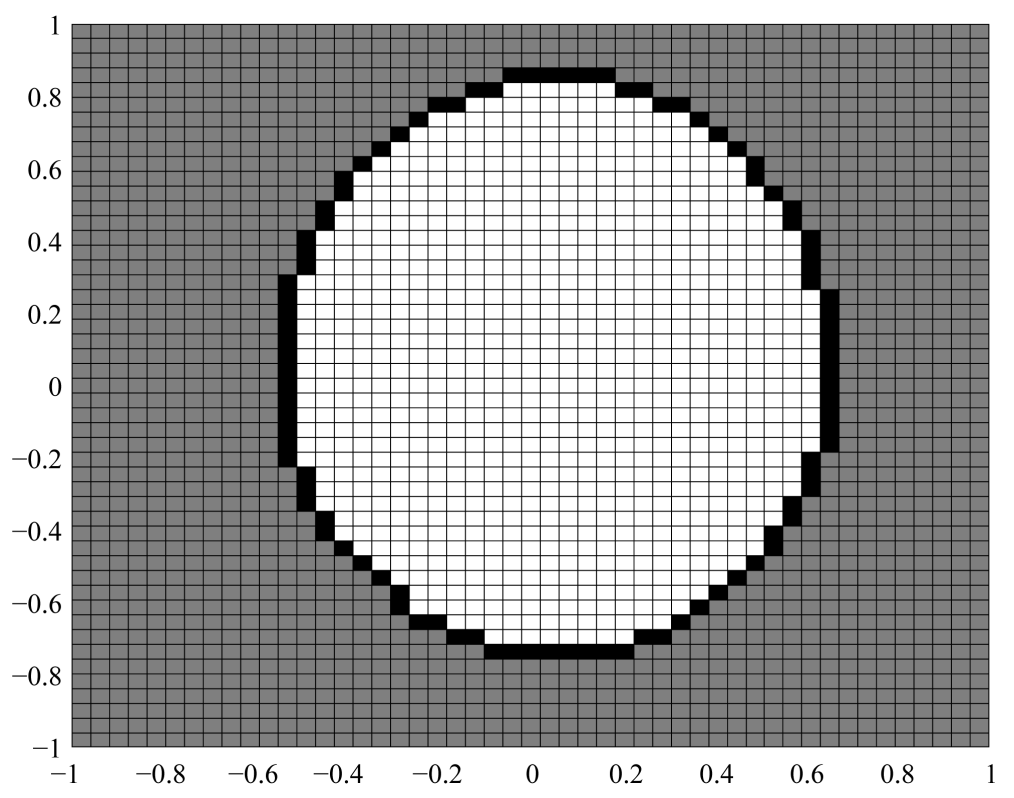

Figure 2. Discretization of studied domain $\Omega \subset[-1,1] \times[-1,1]$ into cells.

Table 1. Numerical extinction time relatively to time iteration parameter $\mathrm{n}$.

\begin{tabular}{ccccccccc}
\hline$n$ & 13,000 & 16,000 & 21,000 & 31,000 & 61,000 & 70,000 & 100,000 & $>500,000$ \\
$T$ & 0.13 & 0.16 & 0.21 & 0.31 & 0.61 & 0.6123 & 0.6123 & 0.6123 \\
\hline
\end{tabular}



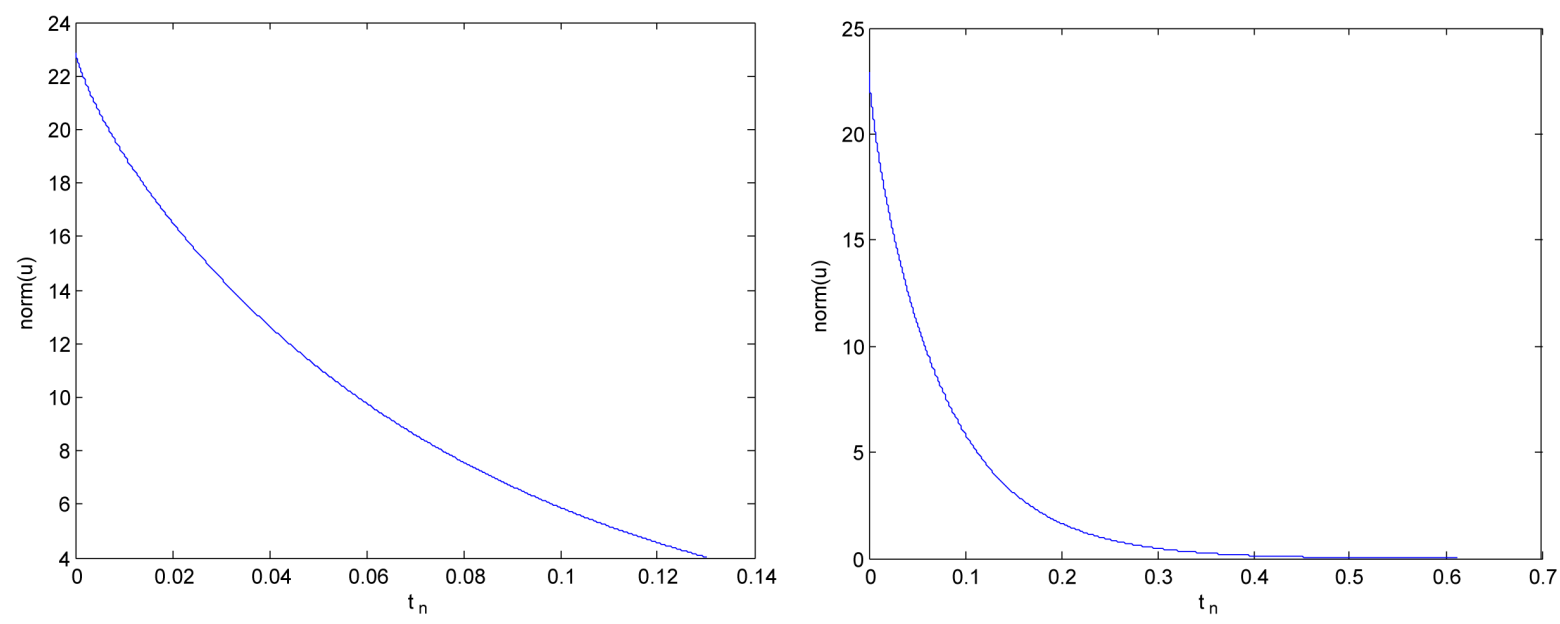

Figure 3. Variation norm of the numerical solution.
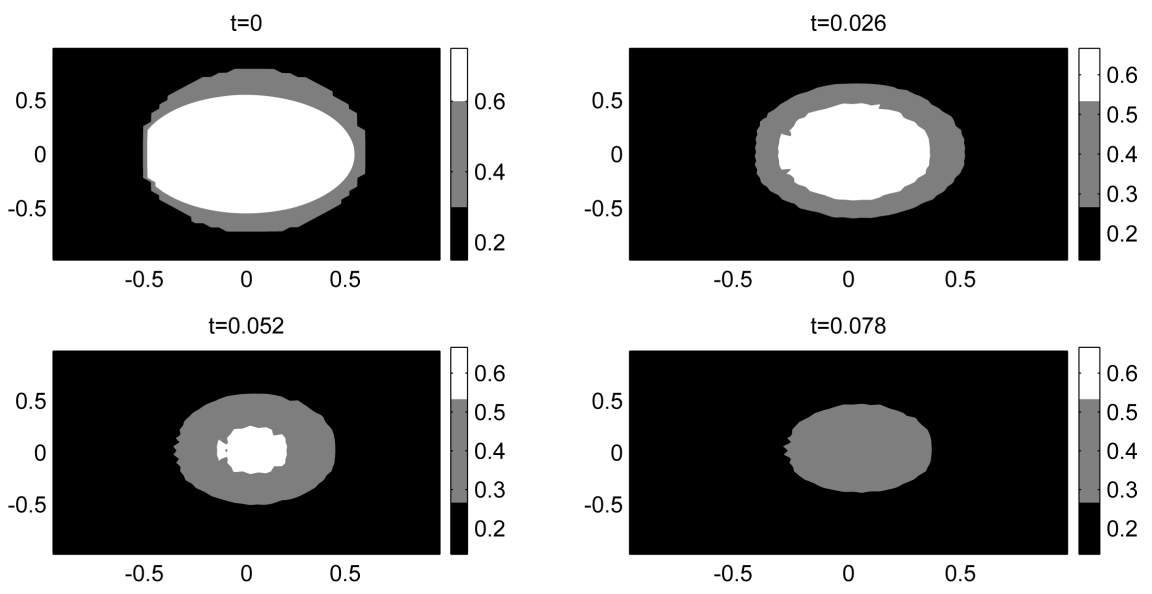

$\mathrm{t}=0.104$

$\mathrm{t}=0.13$
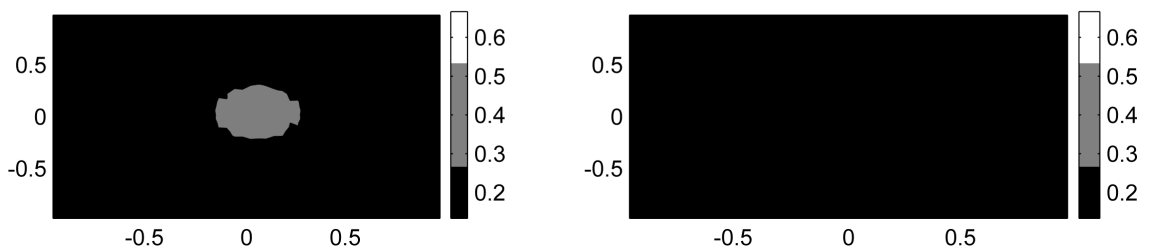

Figure 4. Extinction phenomenon of the numerical solution.

experimentally for a class of nonlinear parabolic equations that the numerical algorithm is efficient for computing the extinction time of solutions.

In the works to come, it will be better to apply the numerical algorithm to study, for example, moving boundary problems and extinction problems in environment.

\section{Acknowledgements}

We thank the Editor and the referee for their comments.

\section{References}

[1] Kalashnikov, A.S. (1974) The Propagation of Disturbances in Problems of Non-Linear Heat Conduction with Absorption. USSR Computational Mathematics and Mathematical Physics, 14, 70-85. 
http://dx.doi.org/10.1016/0041-5553(74)90073-1

[2] Galaktionov, V.A. and Vasquez, J.L. (2002) The Problem of Blow-Up in Nonlinear Parabolic Equations. Discrete and Continuous Dynamics Systems, 8, 399-433. http://dx.doi.org/10.3934/dcds.2002.8.399

[3] Levine, H.A. (1985) The Phenomenon of Quenching: A Servey. North-Holland Mathematics Studied, 110, $275-286$. http://dx.doi.org/10.1016/S0304-0208(08)72720-8

[4] Diaz, J.I. (2001) Qualitative Study of Nonlinear Parabolic Equations: An Introduction. Extracta Mathematicae, 16, 303-341.

[5] Friedman, A. and Herrero, M.A. (1987) Extinction Properties of Semilinear HEAT Equations with Strong Absorption. Journal of Mathematical Analysis and Applications, 124, 530-546. http://dx.doi.org/10.1016/0022-247X(87)90013-8

[6] Gu, Y.G. (1994) Necessary and Sufficient Conditions for Extinction of Solutions to Parabolic Equations. Acta Mathematica Sinica, 37, 73-79.

[7] Lair, A.V. (1993) Finite Extinction Time for Solutions of Nonlinear Parabolic Equations. Nonlinear Analysis, Theory, Methods and Applications, 21, 1-8.

[8] Boni, T.K. (2001) Extinction for Dicretizations of Some Semilinear Parabolic Equations. Comptes Rendus de l'Acadmie des Sciences de Paris, Serie I, Mathmatique, 333, 795-800.

[9] Mikula, K.B. (1995) Numerical Solution of Nonlinear Diffusion with Finite Extinction Phenomenom. Acta Mathematica Universitatis Comenianae, LXIV, 173-184.

[10] Nabongo, D. and Boni, T.K. (2008) Numerical Quenching for a Semilinear Parabolic Equation. Mathematical Modelling and Analysis, 13, 521-538. http://dx.doi.org/10.3846/1392-6292.2008.13.521-538

[11] Nabongo, D. and Boni, T.K. (2008) Quenching for Semidiscretization of a Semilinear Heat Equation with Dirichlet and Neumann Boundary Condition. Commentationes Mathematicae Universitatis Carolinae, 49, 463-475.

[12] Lair, A.V. and Oxley, M.K. (1996) Anisotropic Nonlinear Diffusion with Absorption: Existence and Extinction. International Journal of Mathematics and Mathematical Sciences, 19, 427-434. http://dx.doi.org/10.1155/S0161171296000610

[13] Dumitrache, A. (2007) A Numerical Method to Approximate the Solutions of Nonlinear Absorption Diffusion Equation. Proceeding in Applied Mathematics and Mechanics, 7, 4070041-4070042. http://dx.doi.org/10.1002/pamm.200701050

[14] Kim, D. and Proskurowski, W. (2004) An Efficient Approach for Solving a Class of Nonlinear 2D Parabolic PDEs. International Journal of Mathematics and Mathematical Sciences, 2004, 881-899.

[15] Griebel, M., Dornseifer, T. and Neunhoeffer, T. (1998) Numerical Simulation in Fluid Dynamics. A Practical Guide, SIAM, Philadephia. 